\title{
BMJ Open The outcomes of recent patient safety education interventions for trainee physicians and medical students: a systematic review
}

\author{
Matthew A Kirkman, ${ }^{1}$ Nick Sevdalis, ${ }^{2}$ Sonal Arora, ${ }^{3}$ Paul Baker, ${ }^{4}$ Charles Vincent, ${ }^{5}$
} Maria Ahmed ${ }^{6}$

To cite: Kirkman MA, Sevdalis N, Arora S, et al. The outcomes of recent patient safety education interventions for trainee physicians and medical students: a systematic review. BMJ Open 2015;5:e007705. doi:10.1136/bmjopen-2015007705

\section{- Prepublication history} and additional material is available. To view please visit the journal (http://dx.doi.org/ 10.1136/bmjopen-2015007705).

Received 16 January 2015 Revised 14 April 2015 Accepted 16 April 2015

CrossMark

For numbered affiliations see end of article.

Correspondence to Dr Maria Ahmed; maria.k.ahmed@gmail.com

\begin{abstract}
Objective: To systematically review the latest evidence for patient safety education for physicians in training and medical students, updating, extending and improving on a previous systematic review on this topic. Design: A systematic review.

Data sources: Embase, Ovid Medline and PsycINFO databases.

Study selection: Studies including an evaluation of patient safety training interventions delivered to trainees/residents and medical students published between January 2009 and May 2014.
\end{abstract}

Data extraction: The review was performed using a structured data capture tool. Thematic analysis also identified factors influencing successful implementation of interventions.

Results: We identified 26 studies reporting patient safety interventions: 11 involving students and 15 involving trainees/residents. Common educational content included a general overview of patient safety, root cause/systems-based analysis, communication and teamwork skills, and quality improvement principles and methodologies. The majority of courses were well received by learners, and improved patient safety knowledge, skills and attitudes. Moreover, some interventions were shown to result in positive behaviours, notably subsequent engagement in quality improvement projects. No studies demonstrated patient benefit. Availability of expert faculty, competing curricular/service demands and institutional culture were important factors affecting implementation.

Conclusions: There is an increasing trend for developing educational interventions in patient safety delivered to trainees/residents and medical students. However, significant methodological shortcomings remain and additional evidence of impact on patient outcomes is needed. While there is some evidence of enhanced efforts to promote sustainability of such interventions, further work is needed to encourage their wider adoption and spread.

\section{INTRODUCTION}

Educational interventions for quality and safety improvement have garnered increasing

\section{Strengths and limitations of this study}

- This systematic review provides an update of the evidence on courses teaching core concepts of patient safety to medical students and trainees/ residents.

- The results confirm an increasing trend for developing educational interventions in patient safety delivered to trainees/residents and medical students.

- However, we found that significant methodological shortcomings in studies reporting such interventions remain and additional evidence of impact on patient outcomes is needed.

- While there is some evidence of enhanced efforts to promote sustainability of such interventions, further work is needed to encourage their wider adoption and spread.

- The main limitations of this systematic review relate to the quality of the included studies, and to only including articles published in the English language.

interest over recent years. The importance of such interventions is acknowledged by the development and integration of dedicated patient safety and quality improvement curricula and frameworks into medical education at all levels. For example, the Association of American Medical Colleges (AAMC) endorses the introduction of formal quality improvement education from medical school through to postgraduate training and continuing medical education. ${ }^{1}{ }^{2}$ The Accreditation Council for Graduate Medical Education (ACGME) ${ }^{3}$ and CanMEDS ${ }^{4}$ competency frameworks incorporate essential competencies relating to quality and safety for medical professionals. The WHO has developed a Patient Safety Curriculum Guide for Medical Schools ${ }^{6}$ and, recently, a multiprofessional edition. ${ }^{7}$ Such curricula aim to guide and support educators in developing 
and implementing educational programmes in patient safety.

There has been a significant increase in the number of publications relating to patient safety courses, particularly those aimed at residents. A systematic review on teaching patient safety and quality improvement to medical students and residents was published in 2010, ${ }^{8}$ identifying 41 studies published between January 2000 and January 2009, of which 27 included an evaluation of the described intervention. This review identified significant methodological limitations in most studies, including low response rates, single centre recruitment and small sample sizes (median $=41$ participants per study, IQR 20-106). ${ }^{8}$ Although most interventions were well received by participants, and resulted in improvements in safety and quality knowledge scores, few studies were able to demonstrate changes in learners' behaviour or potential patient benefit. ${ }^{8}$ The reviewed articles also identified multiple barriers to sustainable integration of the courses, which spanned learner, faculty and institutional factors. ${ }^{8}$

Patient safety education is a rapidly emerging field and it is likely that, in part due to the recent development and implementation of patient safety curricula and frameworks highlighted above, an increasing number of articles have been published since this last systematic review, perhaps addressing some of the aforementioned methodological limitations of the older studies. The aim of this study was thus to perform a focused systematic review of research reporting courses that teach core concepts in patient safety, and that target medical students and junior physicians, published since 1 January 2009. We describe the educational content and teaching methods employed, evaluate the learning outcomes achieved and explore factors influencing implementation of these patient safety courses.

\section{METHODS}

\section{Data sources and search strategy}

We prespecified the methods utilised in this systematic review and present them in accordance with PRISMA (Preferred Reporting Items for Systematic reviews and Meta-Analyses) guidelines. ${ }^{9}$ A literature search was performed using the electronic databases of Embase (1996 to 2014 Week 18), Ovid MEDLINE (1996 to April Week 5 2014) and PsycINFO (2002 to May Week 1 2014); although the focus of this systematic review was to identify papers published since the last systematic review covering this topic, that is, from January 2009 onwards, we used a search strategy incorporating earlier start dates. This allowed us to perform an evaluation of the sensitivity of our search strategy by ensuring five reference papers that we identified as being highly relevant studies before performing the literature review ${ }^{10-14}$ were identified by our search strategy. All five reference papers were identified, and thus we were able to begin our search from the end of the data collection period of the previous systematic review covering this topic. ${ }^{8}$
Our search strategy (see online supplementary appendix 1) incorporated the two broad themes of 'medical education' and 'patient safety', and the content areas were combined using the Boolean operator 'and'; a pilot search revealed that 'medical education' successfully encompassed both 'education' as the intervention and 'medical students and/or trainees and/or residents' as the population of interest. Search terms were generated with the assistance of key words from core reference texts ${ }^{15}$ and relevant articles, ${ }^{8}$ and a combination of $\mathrm{MeSH}$ terms and free text words (truncated wherever appropriate) were used to maximise the sensitivity of the search. We limited the search to human studies published in English language, and removed duplicates. Additional articles were sought through hand searching of reference lists of included studies.

As our data comprised studies that were previously published and publicly available, this study did not require ethical approval.

\section{Eligibility criteria}

We included articles that described and evaluated an educational intervention that explicitly exposed medical students and/or trainees/residents to core concepts of patient safety. Articles that included medical students and/or trainees/residents in addition to other population groups were not excluded. To be included, reviewed articles were required to have sufficient empirical data for analysis (eg, conference proceedings were excluded), the educational intervention was required to include patient safety as core content and the study had to include an evaluation of the educational intervention. Detailed eligibility criteria can be found in online supplementary appendix 2 .

\section{Article review process}

Titles of the initial 4027 articles identified by the search strategy outlined above were reviewed by an academic physician with expertise in patient safety and medical education (MA). After excluding articles with titles that were clearly irrelevant to the topic at hand, the remaining abstracts were reviewed for inclusion independently by MA and a second physician with expertise in medical education (MAK). Disagreements were resolved through consensus, involving a third reviewer with expertise in patient safety and medical education (NS), as necessary.

\section{Data extraction and quality assessment}

Consistent with Best Evidence Medical Education (BEME) recommendations, ${ }^{16}$ administrative data (including publication details and country of origin), topic-related data (including details of the educational intervention and number and type of participants) and research-related data (including methodology and results) were extracted from the studies that were identified as relevant. Factors influencing curricular implementation of the intervention were categorised under four broad headings (learner factors, faculty factors, 
curricular factors and learning environment factors) devised by the authors of the previous systematic review on this topic. ${ }^{8}$ Only factors that were explicitly described by the authors of the papers included in this systematic review were counted and categorised in this manner.

Assessing the quality of interventions is a welldocumented challenge facing systematic reviews of educational interventions. ${ }^{17}$ The BEME review protocol recommends a system for assessing the quality of studies based on grading, ${ }^{16}$ but as no specific guidance as to how to apply these grades is provided, we assessed quality by extracting information on both stated and perceived limitations of the study as assessed by study design, sample size, completeness of data and overall coherence between study aims, methods and conclusions.

\section{Analysis}

Given the anticipated heterogeneity in study designs and outcomes as per the previous systematic review on this topic, ${ }^{8}$ quantitative synthesis of the data (ie, metaanalysis) was not performed. Simple quantitative statistics were used to report on educational content, methodologies used, study populations and learning outcomes (where reported).

Studies were categorised by the learning outcomes reported by the authors, using the modified version of Kirkpatrick's levels of evaluation adopted by the BEME collaboration as a grading standard for systematic reviews. ${ }^{16}$ This assesses impact on learners' satisfaction (level 1), changes in learners' attitudes (level 2a), measures of learners' knowledge and skills (level 2b), change in learners' behaviour (level 3), changes to clinical processes/organisational practice (level 4a) and benefits to patients (level 4b). Correspondingly, the results of this systematic review are presented according to the Kirkpatrick learning outcome assessed.

\section{RESULTS}

\section{Selected articles}

The initial yield of the review was 4027 articles retrieved by the search strategy. The subsequent title screen of articles identified 304 potentially relevant titles for the abstract review stage. Independent review of abstracts against the eligibility criteria by two reviewers (MAK, MA) followed by consensus resulted in 61 papers for review. The agreement between the reviewers was excellent ( $\kappa=0.917,95 \%$ CI 0.871 to 0.963 ). Review of the full text identified 25 papers that fully met the eligibility criteria for inclusion. An additional eligible paper was identified from hand searching of relevant reference lists, resulting in 26 papers for analysis. This process is summarised in figure 1 .

\section{Characteristics of included studies and study settings}

Table 1 summarises the main characteristics of the included studies, including study design, participant number and type, and course structure and content.

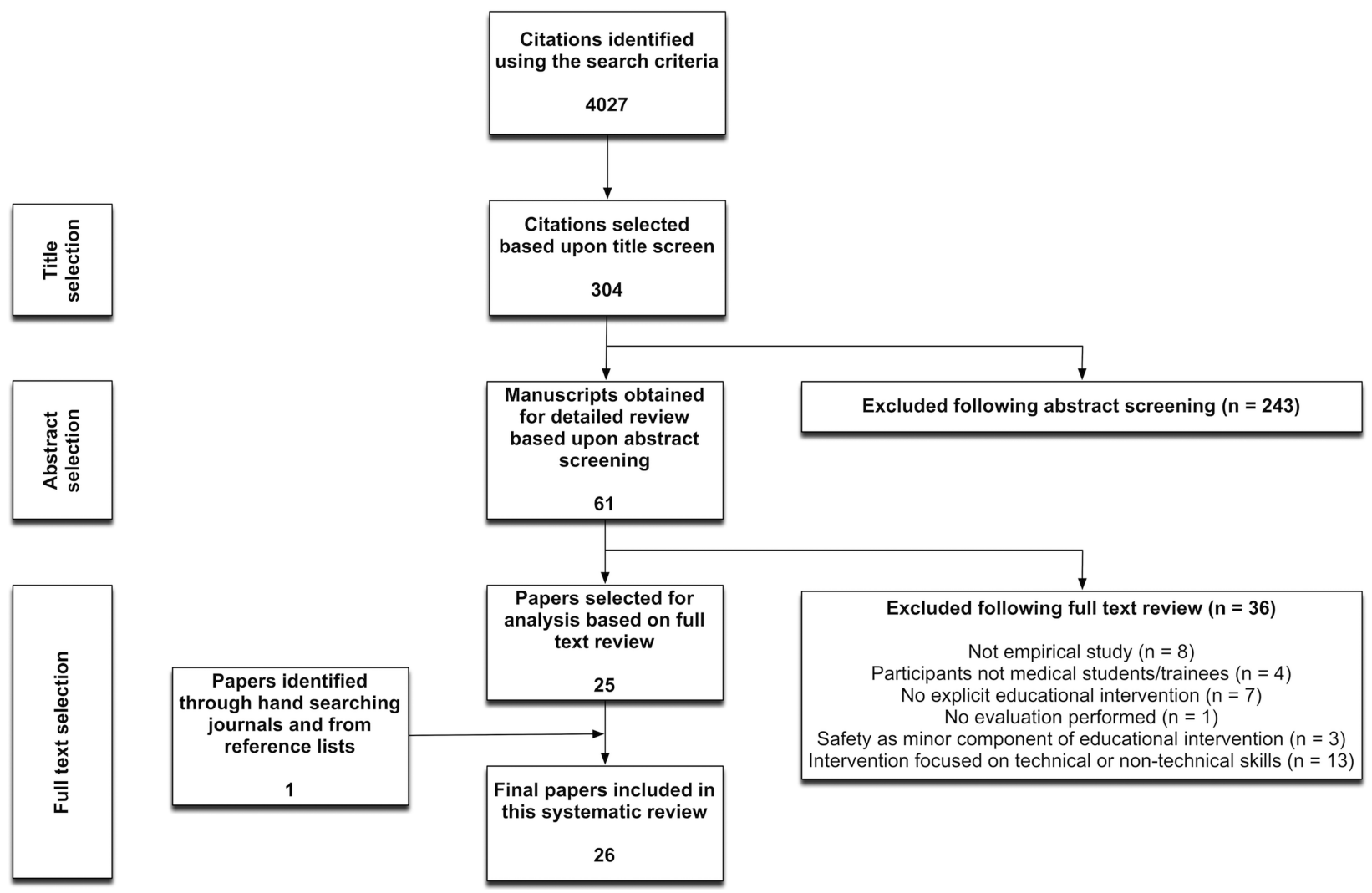

Figure 1 Flow diagram illustrating our search strategy. 
Table 1 Study characteristics, course structure and conten

\begin{tabular}{|c|c|c|c|c|}
\hline $\begin{array}{l}\text { Lead author } \\
\text { Reference } \\
\text { Year } \\
\text { Country }\end{array}$ & Study type & Participant number and specialty & Course structure & Course content \\
\hline $\begin{array}{l}\text { Aboumatar }{ }^{41} \\
2012 \\
\text { USA }\end{array}$ & Before and after study & $\begin{array}{l}120 \text { third-year medical students. Recruited from } \\
\text { a single institution }\end{array}$ & $\begin{array}{l}\text { 3-day clinically oriented patient safety intersession using } \\
\text { role-play and simulation, skills demonstrations, } \\
\text { small-group exercises and case-based learning }\end{array}$ & $\begin{array}{l}\text { Medical error understanding and prevention, } \\
\text { teamwork and communication, systems thinking }\end{array}$ \\
\hline $\begin{array}{l}\text { Ahmed } \\
2014 \\
\text { UK }\end{array}$ & Before and after study & $\begin{array}{l}1169 \text { junior physicians across a region } \\
\text { (16 institutions) }\end{array}$ & $\begin{array}{l}\text { Monthly } 60 \text { min sessions led by junior physicians } \\
\text { between January and July } 2011 \text {. Sessions comprised } \\
\text { case-based discussion and analysis of patient safety } \\
\text { incidents encountered in practice, facilitated by trained } \\
\text { faculty }\end{array}$ & $\begin{array}{l}\text { Key patient safety concepts, RCA/systems-based } \\
\text { analysis, communication and teamwork, incident } \\
\text { reporting }\end{array}$ \\
\hline $\begin{array}{l}\text { Anderson }{ }^{18} \\
2009 \\
\text { UK }\end{array}$ & Before and after study & $\begin{array}{l}199 \text { students including } 58 \text { final year medical } \\
\text { students learning in uni-professional groups } \\
\text { and } 36 \text { learning in interprofessional groups as } \\
\text { part of a regional programme }\end{array}$ & $\begin{array}{l}\text { 1-day workshop involving DVD and small-group } \\
\text { facilitated discussion to analyse key safety issues using } \\
\text { the National Patient Safety Agency RCA tool. } \\
\text { Supporting handbook containing additional relevant } \\
\text { materials. Nine events held over } 2 \text { years }\end{array}$ & $\begin{array}{l}\text { DVD of patient journey to focus on learning themes } \\
\text { of situational awareness, communication, leadershi } \\
\text { and empowerment. RCA }\end{array}$ \\
\hline $\begin{array}{l}\text { Arora }^{21} \\
2012 \\
\text { UK }\end{array}$ & Before and after study & $\begin{array}{l}27 \text { surgical residents. Recruited from across } 19 \\
\text { hospitals }\end{array}$ & $\begin{array}{l}3 \mathrm{~h} \text { training programme comprising lectures, video } \\
\text { demonstrations and small-group discussions }\end{array}$ & $\begin{array}{l}\text { Patient safety overview, adverse events, human } \\
\text { factors, systems-based analysis, communication } \\
\text { and teamwork in surgery }\end{array}$ \\
\hline $\begin{array}{l}\mathrm{Cox}^{28} \\
2009 \\
\text { USA }\end{array}$ & Before and after study & $\begin{array}{l}\text { Over } 787 \text { interprofessional teams of medical, } \\
\text { nursing, health administration and respiratory } \\
\text { therapy students. Recruited from across } 3 \text { sites }\end{array}$ & $\begin{array}{l}\text { 4-week curriculum comprising lectures, problem-based } \\
\text { learning, small-group work, simulation. Participants } \\
\text { given cases describing a medical error. Team-based } \\
\text { simulation of RCA and use of performance improvement } \\
\text { tools. Presentation on completion }\end{array}$ & $\begin{array}{l}\text { Patient safety overview, RCA, QI overview, } \\
\text { teamwork }\end{array}$ \\
\hline $\begin{array}{l}\text { Cox }^{31} \\
2011 \\
\text { USA }\end{array}$ & $\begin{array}{l}\text { Prospective cohort } \\
\text { study }\end{array}$ & $\begin{array}{l}12 \text { faculty members and } 46 \text { internal medicine } \\
\text { residents. } \\
\text { Recruited from a single institution }\end{array}$ & $\begin{array}{l}3 \mathrm{~h} \text { long faculty development session including videos, } \\
\text { role-play and mock facilitation sessions. Plus manual of } \\
\text { key safety education topics. Implementation of an } \\
\text { alternative reporting system for anonymous narratives of } \\
\text { 'care that did not go as intended'. Monthly 'Safety Story' } \\
\text { sessions of } 4-6 \text { residents with faculty member to } \\
\text { discuss contributing factors and propose potential } \\
\text { solutions }\end{array}$ & $\begin{array}{l}\text { Faculty training included patient safety overview, } \\
\text { RCA and teamwork }\end{array}$ \\
\hline $\begin{array}{l}\text { Dudas }^{34} \\
2011 \\
\text { USA }\end{array}$ & $\begin{array}{l}\text { Retrospective pre-post } \\
\text { study }\end{array}$ & $\begin{array}{l}108 \text { medical students (second-year, third-year } \\
\text { and fourth-year students as part of paediatric } \\
\text { clerkship). Recruited from a single institution }\end{array}$ & $\begin{array}{l}\text { During course of 9-week clerkship, } 25 \text { min online video } \\
\text { on systems-based analysis of medical errors. } 60 \mathrm{~min} \\
\text { large-group faculty demonstration of Learning From } \\
\text { Defects tool. Subsequent self-directed small-group } \\
\text { identification and analysis of medication errors in } \\
\text { practice. Group presentation at closing } 60 \text { min session }\end{array}$ & Systems-based analysis \\
\hline $\begin{array}{l}\text { Gupta }^{43} \\
2014 \\
\text { USA }\end{array}$ & $\begin{array}{l}\text { Retrospective pre-post } \\
\text { study }\end{array}$ & $\begin{array}{l}26 \text { neonatology fellows. Recruited from a single } \\
\text { institution }\end{array}$ & $\begin{array}{l}\text { Workshops, web-based modules, completion of a quality } \\
\text { and safety project, presentation at departmental } \\
\text { conference, participation in departmental morbidity and } \\
\text { mortality conference. Optional selected readings and } \\
\text { web-based modules }\end{array}$ & $\begin{array}{l}\text { Core patient safety concepts, QI, human factors, } \\
\text { communication and teamwork, error disclosure, } \\
\text { incident reporting and systems thinking }\end{array}$ \\
\hline $\begin{array}{l}\mathrm{Hall}^{32} \\
2010 \\
\text { USA }\end{array}$ & $\begin{array}{l}\text { Before and after study } \\
\text { and comparison with } \\
\text { historical control }\end{array}$ & $\begin{array}{l}146 \text { third-year medical students undertaking a } \\
\text { medicine clerkship. } 65 \text { in intervention group, } 81 \\
\text { in control group. Recruited from a single } \\
\text { institution }\end{array}$ & $\begin{array}{l}2 \text { mandatory } 1 \mathrm{~h} \text { patient safety 'booster' conferences. } \\
\text { First conference involved RCA brainstorming exercise of } \\
\text { an adverse event. Assignment to identify and } \\
\text { summarise an actual patient safety event or concern. } \\
\text { During second conference case presentation including } \\
\text { proposed system modifications to improve patient safety }\end{array}$ & $\begin{array}{l}\text { RCA including proposed system modifications for } \\
\text { improvement }\end{array}$ \\
\hline
\end{tabular}


Table 1 Continued

Lead author

Reference

Year

Country Study type

Participant number and specialty

\section{Course structure}

Holland $^{42} \quad$ Before and after study

2010

USA

Jansma $^{23}$

2010

The

Netherlands

Jansma $^{24}$

2010

The

Netherlands

Jericho $^{33}$

2010

USA

$\mathrm{Jha}^{22}$

2013

UK

Leung $^{25}$

2010

China

Miller $^{27}$

2014

USA

Myung $^{26}$

2012

Republic of

Korea

Paxton $^{37}$

2010

USA

Rodrigue $^{30}$

2013

USA

Scott ${ }^{40}$

2011

USA

Before and after study with 6-month follow-up

Prospective cohort

study

Before and after study

Before and after study with control group and follow-up

Before and after study

Before and after study 110 medical and allied health students.

Before and after study

Before and after study with control group and

follow-up

Before and after study

Prospective cohort

study
130 third-year medical students. Recruited from a single institution

Recruited from a single institution

156 second-year medical students. Recruited from a single institution 26 PGY-3 internal medical residents. Recruite
from a single institution

33 specialty registrars (GP, anaesthesiology, a single institution

71 residents (surgical and non-surgical). Recruited from 5 hospitals

Anaesthesiology residents (approximately a single institution

263 junior physicians across a region (155 in intervention group, 108 in control group)

51 surgical clerkship students including 46 medical and 5 physician assistant students. Recruited from a single institution

42 residents and 36 faculty members. Recruited from a single institution

680 residents across medical and surgical specialties. Recruited from a single institution 4-week rotation comprising web-based patient safety and QI curriculum including interactive modules, and self-directed reading and assignments. Completion of $Q$ proposal and presentation at end of rotation

2-day course comprising plenaries, group discussions and role-play

Multispecialty 2-day patient safety course including plenaries and small-group sessions. At end of course participants asked to formulate 1 action point to improve patient safety

$90 \mathrm{~min}$ interactive case-based lecture coupled with an expectation of adverse event reporting. Supplemented with education manual. Quarterly conferences to discuss reports and near-immediate feedback from Department of Safety and Risk Management

$3 \mathrm{~h}$ teaching session. Intervention group: patients shared their stories about their experience of safety incidents. Non-intervention group: teaching delivered using "standard methods of teaching", including presentations and small-group work

Two 60 min whole-class lectures using contemporary medical incidents as illustrative cases

$1 \mathrm{~h}$ introductory lecture discussing general patient safety and QI topics followed by 2 courses ('Introduction to the Culture of Safety' and 'Teamwork and Communication') including group discussions

1-week course composed of interactive lecture, discussion and small-group debriefing

$2 \mathrm{~h}$ small-group discussion incorporating slide presentation

5 online modules that residents and faculty members completed together in pairs (duration of each module unreported)

Economic incentive comprised retirement benefit of

$1.5 \%$ of residents' annual salaries. Multifaceted

educational campaign including monthly email notifications, audience presentation at major conferences (exact frequency not stated) and one-on-one discussion

\section{Course content}

Patient safety overview, QI overview including PDSA, medical error, RCA, human factors engineering, safety interventions

Patient safety overview, human error, disclosure, medicolegal aspects of critical incidents, RCA, tips and tools to improve safety in practice

Patient safety overview, human factors, teamwork, contribution to safer care (including RCA),

medicolegal aspects

Patient safety definitions, adverse event reporting, investigation/process improvements,

communication, and apology and remedy

Error analysis. Teaching session covered: prescribing, teamwork and communication

Based on WHO curriculum: patient safety overview, human factors, systems thinking, team working, understanding and learning from error, introduction to QI, medication safety

Patient safety, QI, teamwork, communication

Based on WHO curriculum: patient safety overview, human factors, systems thinking, team working, understanding and learning from error, introduction to $\mathrm{Q}$, medication safety; in addition: RCA Patient safety overview, RCA, epidemiology, error theory, error disclosure and legal considerations

Performance improvement, QI, patient safety, teaching and learning

Presentation covered mechanics of incident reporting, discussing barriers and dispelling myths 


\section{Lead author}

Reference

Year

Country

Study type

Participant number and specialty

Shaw ${ }^{39} \quad$ Randomised controlled 371 interns across medical and surgical

2012 trial specialties. Recruited from across 2 hospitals

USA

Slater ${ }^{19} \quad$ Before and after study

2012

UK

Smith ${ }^{35}$

2012

USA

Stah $^{38} \quad$ Before and after study

$2011 \quad$ with control group

USA

Tess $^{44}$

2009

USA

Wilson $^{29}$

2012

Prospective cohort

USA

study

Prospective cohort

study

11 multiprofessional teams comprising 55

health professionals (including 16 junio

physicians and 12 senior physicians)

Recruited from across 5 sites

280 internal medicine residents over 2 years.

Recruited from a single institution$$
\text { Retrosp }
$$

study

single institution $\mathrm{SQ}$, safety questionnaire; TAPS, Training and Action for Patient Safety.

110 third-year medical students on surgical group). Recruited from a single institution students).

Recruited from a single institution
Course structure

2 interventions compared: Online Spaced Education programme consisting of cases and questions that reinforce over time, and SQ programme comprising online slide-show followed by quiz

20-week 'TAPS' programme. $2 \mathrm{~h}$ online learning module; Human error, Ql tools (process mapping, fishbone multiprofessional workshops to conduct QI project, executive-group discussion for organisational learning

Monthly noontime QIC. RCA of selected real-life safety events (selected by seniors, analysed by residents not associated with the case). Limited RCA with online resources and mentorship. Presentation to fellow residents and seniors. Intervention proposed and followed through where possible clerkship (67 in intervention group, 43 in contro

Two-part patient safety curriculum: all students attended 1-day lecture on introductory theories, video and small-group discussion (first year). Intervention group attended additional $1.5-2 \mathrm{~h}$ clinically oriented classroom discussion, videos, simulation and role-play (third year) Educational intervention coupled with reorganisation of clinical services to integrate patient safety and QI into daily clinical practice. The educational intervention incorporated an online module in year 1, and a 3-week rotation in QI in year 2. Faculty-led workshops on RCA performance improvement and the institutional approach to QI

23 graduate level students (including 7 medica
Course content

Covered all 92009 NPSGs including handover patient identification, hand hygiene and medication safety

Human error, QI tools (process mapping, fishbon
diagrams and measurement for improvement)

RCA and QI

Patient safety principles, crew resource management, team skills, task management and situational awareness

Patient safety overview, QI and RCA

Patient safety overview, human factors analysis, systems approach to error analysis, crew resource management, law, and policy, and team building
Weekly $3 \mathrm{~h}$ sessions held over a 15-week period. Each session comprised a presentation by a visiting expert, discussion on assigned reading material and small-group patient safety project work

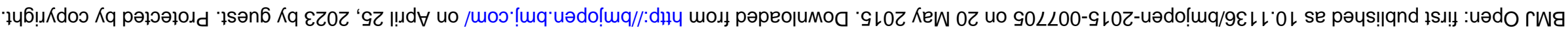


The majority of the 26 studies were conducted in the USA $(n=17,65 \%)$. Of the remaining studies, five $(19 \%)$ came from the UK, ${ }^{18-22}$ two $(8 \%)$ from the Netherlands, ${ }^{23}{ }^{24}$ one from China ${ }^{25}$ and one from the Republic of Korea. ${ }^{26}$ Participants comprised trainees in $15(58 \%)$ studies (often resident or specialty trainee/ registrar grade), and medical students in the remainder. No studies recruited students and trainees/residents simultaneously. Participants learned in interdisciplinary groups in six of the studies: four involved students, ${ }^{18} 27-29$ one included junior and senior physicians, ${ }^{19}$ and another comprised residents and faculty. ${ }^{30}$ One study involved senior physicians (attending or consultant grade level) participating as part of faculty development activities, although their learning outcomes were not directly assessed. ${ }^{31}$

\section{Characteristics of the courses}

Features of the courses including the teaching modalities employed and the core content covered are summarised in table 2. The majority of courses employed a mixture of didactic and experiential teaching methods. Small-group discussions/workshops and lectures were commonly used approaches: $\mathrm{n}=14(54 \%)$ and $\mathrm{n}=12$ (46\%) of courses, respectively. Multimedia approaches including web-based content, videos and/or DVDs were also employed in 10 studies (38\%), mostly as an adjunct to other approaches and less so as a central feature of the course. Case-based learning utilising real-life examples of adverse events identified by either participants themselves ${ }^{20}{ }^{31-35}$ or presented by patients, ${ }^{22}$ was used as a core feature in seven (27\%) courses. Project work (quality or safety improvement) was used in six studies $(23 \%)$, and role-play and simulation were used in only four studies $(15 \%)$. The latter is in contrast to studies of non-technical skills training (such as team training), which typically rely on resource-intensive simulation-based teaching modalities. ${ }^{36}$

The most common content of the courses included a general overview of patient safety (including key terminology and the emergence of patient safety) and root cause analysis and/or systems-based analysis, featured in $17(65 \%)$ and $16(62 \%)$ studies, respectively. Communication and teamwork skills (both core "nontechnical skills') education was included in 13 (50\%) studies, and quality improvement principles and methodologies in $12(46 \%)$ studies. 'Human factors (engineering)' and 'systems thinking' were also covered in some studies, although these phrases were typically illdefined by authors. Other less frequently covered content included medication safety, error disclosure, and incident reporting methods and barriers. Only three studies (12\%) explicitly based their curricular content on the WHO's Patient Safety Curriculum Guide for Medical Schools; interestingly, all three were studies conducted outside of the USA. ${ }^{21} 2526$ Of studies conducted in the USA, nine $(53 \%)$ cited regulatory standards in education as the rationale to their work.

Table 2 Core features of the courses studied, and Kirkpatrick's levels of evaluation

\begin{tabular}{|c|c|c|c|}
\hline Characteristic & $\begin{array}{l}\text { Studies involving } \\
\text { students }(n=11)\end{array}$ & $\begin{array}{l}\text { Studies involving } \\
\text { trainees/residents }(n=15)\end{array}$ & $\begin{array}{l}\text { All studies }(n=26) ; \\
\text { number }(\%)\end{array}$ \\
\hline \multicolumn{4}{|l|}{ Educational modality } \\
\hline Small-group discussion/workshop & 8 & 6 & $14(54)$ \\
\hline Lecture & 7 & 5 & $12(46)$ \\
\hline Multimedia (web, DVD) & 3 & 7 & $10(38)$ \\
\hline Case-based learning & 2 & 5 & $7(27)$ \\
\hline Project/presentation requirement & 2 & 4 & $6(23)$ \\
\hline Simulation/role-play & 3 & 1 & $4(15)$ \\
\hline \multicolumn{4}{|l|}{ Core content } \\
\hline $\begin{array}{l}\text { Patient safety overview (includes } \\
\text { key terminology, emergence of safety) }\end{array}$ & 7 & 10 & $17(65)$ \\
\hline Root cause/systems-based analysis & 6 & 10 & $16(62)$ \\
\hline Communication and teamwork & 6 & 7 & $13(50)$ \\
\hline Quality improvement & 4 & 8 & $12(46)$ \\
\hline 'Human factors' & 2 & 6 & $8(31)$ \\
\hline 'Systems thinking' & 3 & 2 & $5(19)$ \\
\hline Medication safety & 2 & 2 & $4(15)$ \\
\hline Error disclosure & 1 & 3 & $4(15)$ \\
\hline Incident reporting (methods, barriers) & 0 & 3 & $3(12)$ \\
\hline \multicolumn{4}{|l|}{ Kirkpatrick's level of evaluation } \\
\hline 1: Participation & 7 & 12 & $19(73)$ \\
\hline 2a: Attitudes/perceptions & 9 & 11 & $20(77)$ \\
\hline 2b: Knowledge/skills & 7 & 7 & $14(54)$ \\
\hline 3: Behavioural change & 3 & 13 & $16(62)$ \\
\hline 4a: Organisational change & 0 & 6 & $6(23)$ \\
\hline 4b: Patient benefit & 0 & 0 & 0 \\
\hline
\end{tabular}


This included reference to the AAMC Medical Schools Objective Project report, which recommends that medical schools deliver patient safety education to undergraduates, ${ }^{1}$ and the ACGME, ${ }^{3}$ which lists common competencies in practice-based learning and systems-based practice.

\section{Study design and quality assessment}

The majority of studies employed a before-and-after study design $(n=18,69 \%)$; four of these included a control group: two involved a contemporaneous control, ${ }^{22} 37$ one a historical control, ${ }^{32}$ and one a randomised contemporaneous control group. ${ }^{38}$ Only three (12\%) studies included additional long-term follow-up, at 6 weeks, ${ }^{22} 6$ months, ${ }^{23}$ or 'between 1 and 12 months' ${ }^{37}$ Five (19\%) studies involved a postintervention evaluation only. One study was a randomised controlled trial, however, due to logistical constraints, the control group did not undergo matched assessment of behavioural outcome measures. ${ }^{39}$

The median sample size across studies was 109 participants (IQR 52-188), and one outlier study had 1169 participants; ${ }^{20}$ some studies did not clearly indicate the exact number of participants. For example, one study was described as involving 'over 787' participants pooled over several years. ${ }^{28}$ The majority of studies were conducted within a single institution $(\mathrm{n}=18,69 \%)$. Other common methodological limitations included poor response rates, ${ }^{19} 22354041$ inadequate description of the course $^{19} \quad 39$ and/or inadequate reporting of results. ${ }^{22} 282933$ Limitations relating to the assessment tools employed are described in the following section.

\section{Study evaluation and main findings}

Table 2 displays the levels of evaluation assessed across the studies categorised by participant type (medical student or trainee/resident). Studies involving students primarily focused on participant satisfaction, attitudes and knowledge/skill acquisition, with less emphasis on behavioural change. In contrast, nearly all $(n=13$ of 15 , $87 \%$ ) studies involving trainees/residents examined behavioural change as a learning outcome, with six (23\%) studies examining organisational impact through participant engagement in quality improvement work. $^{19} 203542-44$ None of the studies explored patient benefit (level $4 \mathrm{~b}$ ) as a result of the course.

The outcome measures, main findings and level(s) of evaluation reported in each study are displayed in table 3 . Assessment tools used and main findings are discussed further under the respective Kirkpatrick's level headings below.

\section{Level 1: participation/satisfaction}

This was assessed in $19(73 \%)$ studies. Satisfaction was mostly assessed using questionnaires postintervention requiring responses on a Likert scale. Three studies supplemented satisfaction questionnaires with either focus groups ${ }^{18}{ }^{39}$ or interviews with participants. ${ }^{19}$ Satisfaction with the courses was generally high, although response rates were poor in some studies. ${ }^{22} 3541$ Two studies evaluating courses that included web-based content reported poor uptake ${ }^{34}$ or lower satisfaction rates ${ }^{19}$ with the web-based learning component.

\section{Level 2a: attitudes/perceptions}

Patient safety attitudes/perceptions were assessed using a variety of tools in $20(77 \%)$ studies. Bespoke questionnaires comprising items mapped to course learning

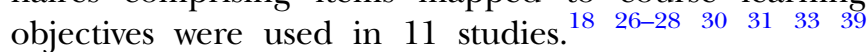
404244 Two studies used modified versions of validated tools, ${ }^{21}{ }^{34}$ and a further four studies used modified versions of previously published questionnaires. ${ }^{20} 232532$ One study used the previously published 'Attitudes to Patient Safety Questionnaire'. ${ }^{22}$ One study assessed systems-based thinking using a validated scale ('System Thinking Scale', STS), ${ }^{41}$ and one study assessed perceived patient safety culture using the modified 'Hospital Survey on Patient Safety Culture'. ${ }^{19}$ Of studies evaluating patient safety attitudes preintervention and postintervention, the majority of studies reported significant improvement in at least some domains. The study assessing systems-based thinking reported significant improvement in STS scale scores postintervention, ${ }^{41}$ while the study evaluating perceived patient safety culture reported no change postintervention. ${ }^{19}$

\section{Level 2b: knowledge/skill acquisition}

Fourteen (54\%) studies evaluated knowledge acquisition using objective and/or self-report measures. Objective tests were used in 12 studies; ${ }^{18-22} 3137-3941-43$ these comprised multiple choice or true/false questions mapped to course learning objectives. One of these studies used knowledge questions from the 'Attitudes to Patient Safety Questionnaire'.22 Most studies demonstrated significant improvements in knowledge acquisition, although in one study a poor response rate precluded statistical testing, ${ }^{19}$ in another no comparison between preintervention and postintervention scores was reported, ${ }^{43}$ and in yet another, increases in performance were observed postintervention but no statistical analyses were reported. ${ }^{22}$

Learners' patient safety skills were assessed in seven (27\%) studies, ${ }^{20} 28323941-43$ all of which employed selfreported measures. Six of these studies demonstrated significant improvement in scores for most or all items, with the remaining study not reporting a comparison between preintervention and postintervention scores. ${ }^{43}$

\section{Level 3: behavioural change}

Changes in safety-related behaviours were assessed in 16 $(62 \%)$ studies, in a number of ways: behavioural intentions assessed via questionnaire ${ }^{23}{ }^{4}{ }^{4}{ }^{42}$ self-reported safety-related actions (eg, incident reporting); $;^{19} 20$ 22-24 303738 or by safety-related actions determined objectively. ${ }^{20} 2133 \quad 35394044$ Of these latter studies, objective assessment included qualitative assessment of patient safety observations, ${ }^{21}$ National Patient Safety Goal 
Table 3 Study outcome measures and main findings

\begin{tabular}{|c|c|c|c|}
\hline $\begin{array}{l}\text { Lead author } \\
\text { Reference } \\
\text { Year }\end{array}$ & Outcome measures & Main findings & $\begin{array}{l}\text { Level of } \\
\text { evaluation }\end{array}$ \\
\hline $\begin{array}{l}\text { Aboumatar }{ }^{41} \\
2012\end{array}$ & $\begin{array}{l}\text { Primary outcome measures: preintervention and postintervention safety } \\
\text { knowledge (19-item bespoke test), self-efficacy in safety skills (9-item } \\
\text { bespoke survey), system-based thinking (using validated STS). Secondary } \\
\text { outcome measures: Postintervention student satisfaction and safety } \\
\text { intentions (2-item survey) }\end{array}$ & $\begin{array}{l}\text { High participant satisfaction-intersession quality rated as excellent or very } \\
\text { good by } 92 \% \text {. Significant improvement in composite systems thinking scores } \\
(61.15-67.56, p<0.001) \text {. Significant improvement in self-efficacy for all taught } \\
\text { communication and safety skills ( }<<0.001) \text {. Significant improvement in safety } \\
\text { knowledge scores pre-post }(64 \% \text { vs } 83 \%, p<0.001) \text {. High self-reported safety } \\
\text { behavioural intentions-85\% reported they would speak up about safety } \\
\text { concerns }\end{array}$ & $1,2 a, 2 b, 3$ \\
\hline $\begin{array}{l}\text { Ahmed }^{20} \\
2014\end{array}$ & $\begin{array}{l}\text { Participants' satisfaction postcourse. Patient safety knowledge (MCQs), skills } \\
\text { (bespoke questionnaire) and safety attitudes (modified validated } \\
\text { questionnaire) pre-post. Behavioural change via questionnaire and review of } \\
\text { 'quality improvement databases' }\end{array}$ & $\begin{array}{l}\text { High participant satisfaction. Significant improvement in } 2 \text { of } 4 \text { safety attitudes } \\
\text { domains (ability to influence safety and behavioural intentions). Significant } \\
\text { improvement in objective safety knowledge }(51.1-57.6 \%, p<0.001) \text {. Trainees } \\
\text { reported significantly more patient safety incidents in the } 6 \text { months following } \\
\text { introduction of the intervention ( } p<0.001) \text {. } 32 \text { Ql projects in various stages of } \\
\text { implementation }\end{array}$ & $\begin{array}{l}1,2 a, 2 b, 3 \\
4 a\end{array}$ \\
\hline $\begin{array}{l}\text { Anderson }{ }^{18} \\
2009\end{array}$ & $\begin{array}{l}\text { Multimethod evaluation. Pre-post questionnaire assessing safety knowledge } \\
\text { and perceptions of course (hopes, concerns and expectations). Additional } \\
\text { postcourse satisfaction questionnaire and focus groups }\end{array}$ & $\begin{array}{l}\text { Majority }(>50 \%) \text { satisfied with course; however, low scores on perceived } \\
\text { preparation for the course. Postcourse medical student concerns emerged as } \\
\text { being unfounded, and hopes and expectations in both the uni-professional } \\
\text { and interprofessional groups were met. Focus group revealed consensus of } \\
\text { added value in working interprofessionally. Significant improvement in } \\
\text { students' knowledge whether working uni-professionally or interprofessionally } \\
(p=0.001)\end{array}$ & $1,2 a, 2 b$ \\
\hline $\begin{array}{l}\text { Arora }^{21} \\
2012\end{array}$ & $\begin{array}{l}\text { Participant satisfaction postcourse. Patient safety knowledge (MCQs) and } \\
\text { safety attitudes (modified validated questionnaire) pre-post. Safety event } \\
\text { identification and reporting } 6 \text { months postcourse via proforma }\end{array}$ & $\begin{array}{l}\text { High participant satisfaction-overall satisfaction mean } 4.63 / 5 \text {. Significant } \\
\text { improvement in } 2 \text { of } 4 \text { safety attitude domains (attitudes to error analysis and } \\
\text { improving safety, and ability to influence safety). Significant improvement in } \\
\text { objective safety knowledge ( } 45.3-70.6 \%, p<0.01 \text { ) and subjective safety } \\
\text { knowledge }(p<0.01) \text {. Postcourse, participants recorded a higher number of } \\
\text { observations associated with greater understanding, recognition and analysis } \\
\text { of patient safety issues }\end{array}$ & $1,2 a, 2 b, 3$ \\
\hline $\begin{array}{l}\text { Cox }^{28} \\
2009\end{array}$ & $\begin{array}{l}\text { Professional group differences in attitudes and skills on } 6 \text { subscales (human } \\
\text { fallibility, disclosure of medical errors, teamwork/communication, event } \\
\text { reporting, systems of care, curricular time spent with other professionals). } \\
\text { Assessed by bespoke survey pre-post intervention }\end{array}$ & $\begin{array}{l}\text { Significant professional group differences preintervention in all } 6 \text { subscales. } \\
\text { Postintervention differences in } 4 \text { subscales were resolved with the exception } \\
\text { of human fallibility }(p<0.001) \text { and curricular time spent together }(p<0.001) \text {. } \\
\text { Medical students scored significantly worse on all subscales apart from } \\
\text { human fallibility }\end{array}$ & $2 a, 2 b$ \\
\hline $\begin{array}{l}\text { Cox }^{31} \\
2011\end{array}$ & $\begin{array}{l}\text { Satisfaction via simple survey. Qualitative analysis of narratives using } \\
\text { constant comparative method }\end{array}$ & $\begin{array}{l}\text { High participant satisfaction- }-85 \% \text { rated it as a positive learning experience. } \\
44 \% \text { self-reported improvement in safety attitudes. High participant } \\
\text { engagement- } 78 \% \text { of residents submitted a story and } 87 \% \text { attended at least } \\
1 \text { safety session. } 79 \text { narratives submitted by residents over } 3 \text { months. Majority } \\
\text { of stories involved errors }(86 \%)\end{array}$ & $1,2 a$ \\
\hline $\begin{array}{l}\text { Dudas }^{34} \\
2011\end{array}$ & $\begin{array}{l}\text { Participant satisfaction. Patient safety attitudes (modified items derived from } \\
\text { Safety Attitudes Questionnaire) }\end{array}$ & $\begin{array}{l}\text { High participant satisfaction- }-76 \% \text { recommended that the session continue. } \\
\text { Significant improvements in patient safety attitudes pre-post in } 9 \text { of } 10 \text { items } \\
(p<0.01)\end{array}$ & $1,2 a$ \\
\hline
\end{tabular}


Gupta $^{43} \quad$ Participant satisfaction postcourse (survey). Self-assessment and knowledge assessment about quality and safety principles precourse using a bespoke tool

High participant satisfaction. Experiential components were felt to be of most

aluation

Almost half (49\%) of items in the knowledge assessment were answered correctly preintervention (but no postintervention comparison data were reported). $75 \%$ of participants had ongoing formal or informal roles in QI or patient safety within their current practice environment following the course (specific time postintervention unreported by authors)

Hall ${ }^{32} \quad$ Patient safety attitudes and self-reported safety skills (previously published

2010 tool). Comparison preintervention and 1 year postintervention and with historical control. Analysis of student-submitted reports compared with contemporaneous reports from patient safety reporting system (PSN)

Holland ${ }^{42}$

2010

Jansma $^{23} \quad$ 11-item questionnaire exploring attitudes, intentions and behaviour towards 2010

Jansma $^{24}$ 2010

Jericho $^{33}$ 2010

$\mathrm{Jha}^{22}$

2013

Curriculum evaluation. Objective knowledge assessed via MCQs and true/ false items precourse and immediately postcourse. Reflection on learning assessment at year-end including knowledge, skills, abilities and beliefs items reporting incidents (using vignettes and modified previously published tool). Assessed at baseline, immediately postcourse and 6 months postcourse

Satisfaction and patient safety behaviours (via semistructured interview) 3 months postintervention to assess whether action implemented and the barriers and promoters to action(s)

Attitudes towards adverse event reporting assessed preintervention and postintervention using a bespoke questionnaire (12 months). Quarterly adverse event reports submitted by residents

Acceptability of the intervention by participants postintervention.

Preintervention and postintervention administration of the APSQ, assessing attitudes and knowledge. ${ }^{52}$ Follow-up at 6 weeks: repeat APSQ, in-depth interviews, and an online survey about success in implementing learning points

Patient safety attitudes and self-report knowledge (adapted previously published questionnaire) assessed precourse and 3 months postcourse
At baseline, no differences in any patient safety attitudes or safety skills between intervention and control. At 1 year postcourse, intervention group expressed significantly higher comfort level in identifying the cause for an error postintervention (3.72 vs $3.27, p<0.05$ ). No significant difference in PSN worthy reports or in blame tone between participants and PSN reporters. Significantly higher robustness of proposed solutions by participants compared with PSN reporters ( 3 vs $0, p<0.001$ )

High satisfaction with curriculum (mean 3.53/4). Residents perceived significant improvements in knowledge, skills, abilities, beliefs and commitment to improve quality of care (all $p<0.001)$. Significant improvement in knowledge $(19.50-23.00, \mathrm{p}<0.05)$. 20 QI projects proposed, $50 \%$ at various stages of implementation

Attitudes towards incident reporting significantly improved (5 of 6 vignettes), $p<0.001$. Intentions towards incident reporting significantly improved between baseline and 6 -month follow-up $(p<0.05)$. No significant improvement in reporting behaviour

High participant satisfaction-mainly positive reaction by $67 \%$. 91 action points formulated by 68 participants. $62(90 \%)$ residents reported taking action at 3 months; $50(55 \%)$ actions were carried out fully. Barriers to implementing actions mentioned more than twice as frequently as compared with promoters. Barriers mostly related to work pressures and rotations Significant improvement in attitudes towards reporting (no $p$ value). Number of reports increased from 0 per quarter in the 2 years preintervention to 28 per quarter for the 7 quarters postintervention, with no sign of decay Response to patient involvement in teaching was largely positive. Mean attitude and knowledge scores on the APSQ increased postintervention compared with preintervention (no $p$ values reported). Response rate to 6 -week follow-up APSQ was poor (38\%). Only 6 participants participated in follow-up in-depth interviews; 3 provided evidence of implementation of learning in practice

Participants supportive of inclusion of patient safety in curriculum and in professional examinations. Significant improvement in 8 of 15 items on patient safety attitudes. Significant improvements in all 5 items on self-reported patient safety knowledge; however mean scores still perceived as 'fair' or 'poor'
$1,2 a, 2 b, 3$, 

postintervention

Myung ${ }^{26} \quad$ Participant satisfaction (method not described). Patient safety awareness 2012 (40-item bespoke questionnaire) pre-post

Overall positive feedback about the course content. $69 \%$ of medical students preferred taking the course individually (the remainder preferring a groupwork format). Significant improvement in all items of the survey $(p<0.05)$ assessing patient safety attitudes among medical students

Student and faculty commented on repetition of some material and desire for more interactive educational methods. Significant improvement in patient safety awareness in 36 of 40 items $(p<0.05)$

Paxton $^{37}$

Patient safety knowledge assessed via MCQ precourse and postcourse, and again at between 1 and 12 months postcourse. Application of learning assessed on long-term follow-up. Control group compared precourse and 6 months postcourse

Perceptions of experience with faculty development opportunities,

Rodrigue ${ }^{30}$

2013

performance and $\mathrm{Q}$ tools and training (bespoke survey). Resident

participation in performance improvement, QI and patient safety programmes

$\operatorname{Scott}^{40}$

Satisfaction with reporting mechanism. Participant attitudes and motivation regarding reporting and intervention (bespoke survey). Percentage of all adverse event reports submitted by residents via electronic reporting system

Programme satisfaction using 7-item survey postintervention and focus group to explore experiences. NPSG-knowledge improvement using MCQ test preintervention and postintervention. NPSG-compliant behaviours in a simulation scenario. Self-reported confidence in safety and quality (bespoke survey)

Satisfaction questionnaire to evaluate online module and each workshop. Patient safety culture assessed using modified 'Hospital Survey on Patient Safety Culture' precourse and postcourse. Knowledge assessed using MCQs pre-post. Project outcomes using run charts. Interviews to explore experiences with TAPS

Significant improvement in knowledge score at short-term (29.3-73.7\%, $\mathrm{p}<0.001)$ and long-term follow-up $(49.1 \%, \mathrm{p}<0.001) .57 .1 \%$ said they had applied the information learned in practice. No significant difference in knowledge found in control group

Non-significant increase in number of residents that felt their training programme provided tools and training in QI. Postintervention, residents reported a non-significant increase $(12.1 \%)$ in participation in departmental/ institutional QI or safety projects, with faculty reporting a significant increase $(38.2 \%, p=0.001)$

$83 \%$ felt the system was burdensome. Monthly average number of adverse events reported by residents significantly increased by 5.5 times $(6(1.6 \%)$ to $33(9 \%), p<0.001)$. Significant improvement in relative proportion of near-miss reports $(0.3(6 \%)$ to $9(27 \%), p<0.001)$. Main motivators for reporting were patient wellness $(87 \%)$ and financial incentive $(64 \%)$

Spaced Education participants found cases authentic, engaging and memorable. Significantly higher proportion of Spaced Education interns responded positively to satisfaction and self-reported confidence items ( 4 of 7 items, $\mathrm{p}<0.05)$. Both online programmes significantly improved knowledge $(\mathrm{p}<0.001)$. No significant difference in knowledge in control group. Higher proportion of Spaced Education participants with improved NPSG-behaviours (mean $4.79 / 13$ vs $4.17 / 13$ in SQ group; significant for surgical participants: 5.67 Spaced Education group vs $2.33 \mathrm{SQ}$ group, $\mathrm{p}<0.05$ )

High rates of satisfaction for workshops (mean score 4.1/5), less so for online module (3.3). No change in safety culture scores for most dimensions apart from significant improvement in 'communication/openness' $(p<0.01)$. Improved multiprofessional communication and teamwork reported via interview. Of the 5 participants who completed pre-post knowledge test, all but 1 improved score. 8 of 11 teams demonstrated improvements in patient safety practices/outcomes via run charts

Satisfaction questionnaire to cohorts across the 2 years. Qualitative analysis of cases presented, interventions proposed and success of follow-through

High participant satisfaction—overall quality of QI conference mean 4.49/5 46 interventions suggested; attempt to initiate $25(54 \%)$ and of these 18

(72\%) deemed successful: 8 led to objective permanent system-wide change and 10 resulted in subjective behavioural change

Participant satisfaction. Participant knowledge pre-post (24-item

(number of times observed and intervened in a patient safety risk) 


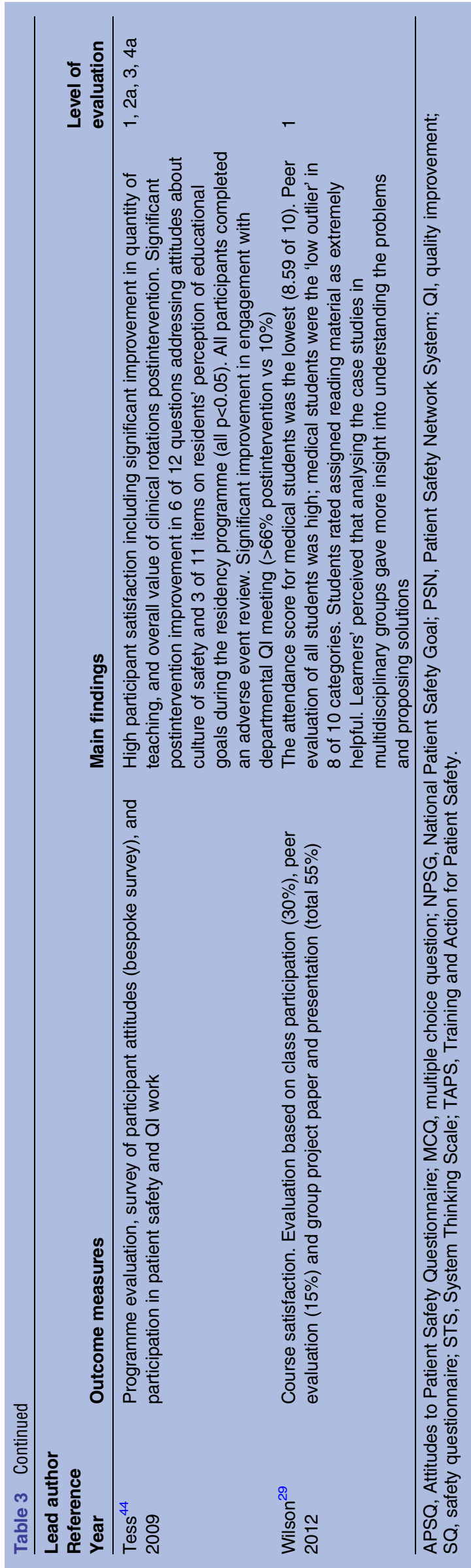

(NPSG)-related behaviours assessed via simulation, ${ }^{39}$ engagement in quality improvement work $^{20} 3544$ and incident reporting assessed via submissions to formal hospital reporting systems. ${ }^{33}{ }^{40}$ All studies reported favourable changes in safety-related behaviours, with the exception of one study, which found that whereas learners' intentions to report significantly improved postcourse, actual (self-reported) incident reporting did not increase following the course. ${ }^{23}$ Notably, all but 3 of the 16 studies that evaluated change in participant behaviour were conducted on trainees/residents as opposed to on medical students.

\section{Level 4a: organisational change}

Six (23\%) studies evaluated organisational change as an outcome measure of their course. Each of these studies involved learner engagement in quality improvement work, ${ }^{19} 203542-44$ and all these studies reported subsequent positive impact at organisational level, including through the initiation/continuation of quality improvement projects/roles. ${ }^{20} 35{ }^{42-44}$ Three quarters of the participants in one study indicated they had a formal or informal role in patient safety or quality improvement within their current practice environment. ${ }^{43}$ The teambased 'Training and Action for Patient Safety' (TAPS) programme found that 8 of the 11 interdisciplinary teams were able to demonstrate improvements in patient safety outcomes and/or practices through the use of weekly data plotted on run charts. ${ }^{19}$

\section{Factors influencing curricular implementation}

Table 4 displays the key factors influencing curricular implementation that we identified, with selected illustrative quotes and categorised under previously designed framework headings. ${ }^{8}$ In terms of learner factors, many studies identified the need to ensure personal/clinical relevance of the material to learners, with opportunities to apply the learning in order to enhance engagement. ${ }^{20} 39$ For studies involving physicians, competing clinical commitments were identified as barriers to engagement. $^{21}$ In studies employing interprofessional modalities, improved teamwork and communication were welcome additional benefits of the course. ${ }^{19}$ However, difficulties in delivering such interprofessional learning were highlighted. ${ }^{28}$ Most studies identified the need for adequate faculty, with protected time to support delivery of the course and competing clinical commitments of faculty being barriers to faculty engagement. ${ }^{20}$ Some commented on the newfound maturity of the faculty infrastructure, ${ }^{41}$ while others aspired to broaden their faculty infrastructure to ensure sustainability of the course. ${ }^{42}$ Faculty role-modelling and clinical credibility were noted to be important influencing factors. ${ }^{25}$

Competing curricular demands were commonly cited as barriers to sustainability of the courses, with some suggesting instituting the course as a mandatory requirement to ensure protected time for learning. ${ }^{21}$ 
Table 4 Factors influencing implementation of patient safety courses

\section{Factors}

Illustrative quotes from published articles

\section{Learner factors}

Enhancing learner engagement by ensuring clinical relevance

Empowering learners through application of learning

Competing clinical/service delivery commitments

Learning interprofessionally improved teamwork and communication

Faculty factors

Investment in faculty development is essential

Faculty role-models and importance of clinical credibility

Protected faculty time

\section{Curricular factors}

Promoting patient safety as a science

Competing curricular demands

Balance between didactic and experiential learning

Balance between reinforcement of learning and repetition of teaching material

Central administrative support necessary for sustainability

Creating interprofessional learning opportunities is challenging Learning environment factors Institutional culture as key to implementation

Ensuring a safe learning environment

Forging improved links between training programmes and hospital improvement activities

Financial support to fund the programme
"The cases, exploring incidents that were largely based on events that had in fact happened, were felt to be realistic and directly applicable to the context of the interns." ${ }^{39}$ "We believe that using authentic clinical scenarios brought forward by trainees as opposed to hypothetical scenarios as in previous studies ensured relevance to trainees and furthermore, stimulated trainee engagement in QI work."20

"Our program challenges residents to apply their skills in systems-based practice to a resident-driven, hospital-based project in an effort to solidify their commitment to QI beyond the structured rotation." 42

"Although all general surgical residents were invited, just more than one half actually attended, citing scheduling conflicts and service delivery pressures as reasons for not doing so."21

"The programme promoted better multi-professional communication and teamwork."19

"Successful implementation of this curriculum, however, requires attention to faculty development. It took several years at our institution to achieve this and some schools may not have similar resources." 41

"Faculty had clinical background and we feel that our students can relate to them more readily." 25

"The residency program further invested in quality by naming both an assistant and associate program director for quality amounting to roughly 0.1 full-time equivalent spent working on the QICs and subsequent project implementation." ${ }^{35}$

"The main barrier to faculty engagement.... was competing clinical commitments." 20

"The topic of safety was approached as a 'science' with a defined set of principles and theories, and supported with published literature." ${ }^{41}$

"Whole-class lectures are by no means the best way to teach patient safety but we find it the easiest format to integrate into a busy curriculum." 25

"Making the course mandatory would have been one way of overcoming this but this would require curricular change at regional level." 21

"The students want to increase small-group discussions and simulation sessions, which would be more effective than didactics." 26

"The rapid decline in long-term post-test scores indicates that...students would benefit from frequent reinforcement of the application of this material." ${ }^{37}$

"Only half of the students elected to view it (online video)...this may be due to the perceived redundancy of the information presented." 34

"We were able to arrange small-group sessions for the randomised, decentralized project for three months, but a core educational activity that includes all residents and is managed centrally would be more sustainable." 31

"It is complicated and time-intensive to plan and deliver meaningful and satisfying inter-professional learning experiences." ${ }^{28}$

"It is important to focus not only on individual attitudes and intentions, but also on a stimulating environment, including hospital culture and patient safety policies." 23

"Several residents commented that they felt safe with the reporting methodologies and follow-up."33

"We believe that few of these reports of safety concerns would have been brought forward without providing a structured forum for discussion in a trusted and collegial environment." 32

"To foster engagement and sustainability, we are now working to more deliberately and consistently integrate patient safety education with the hospitals' systems improvements." 31

"The chair of the department and the program director were very supportive of this endeavour." 35

"VA hospital's willingness to financially support 2 residents per month in this intensive patient safety and quality improvement rotation...Dedicated faculty rotation leaders supported by the VA with protected time to teach and mentor residents." ${ }^{42}$

QI, quality improvement; QICs, QI conferences; VA, Veterans Affairs. 
Promoting patient safety as a science was felt to be a key factor for successful implementation by the authors of one study. ${ }^{41}$ The majority of studies appreciated the need to strike a balance between didactic and experiential teaching modalities, and of the need for sufficient reinforcement while avoiding repetition and duplication of material. The authors of one study recognised that delivering a centrally administered intervention to the whole trainee population may ensure greater sustainability of the course than delivering it to a sample of the cohort. $^{31}$

In terms of institutional/learning environment factors, many studies recognised institutional patient safety culture as a key determinant of successful implementation. ${ }^{23}$ Ensuring a safe learning environment to allow open discussion of sensitive material (eg, relating to adverse events) was recognised as being of particular importance when delivering education on patient safety. Forging improved links between the service provider (hospital) and the training providers was recognised as key to ensuring sustainability, particularly for courses that aimed for engagement in quality improvement work as a follow-on to the course. ${ }^{35}$

\section{Sustainability}

Six $(23 \%)$ studies identified in this review reported data from courses that had been sustained over at least 2 years, ${ }^{18} \quad 2027 \quad 31 \quad 35 \quad 43$ two studies reported 'booster' courses designed to enhance/reinforce established safety educational interventions delivered earlier in the course of training, ${ }^{32} 38$ and one study described an educational intervention coupled with reorganisation of clinical services to facilitate quality and safety improvement efforts. ${ }^{44}$

\section{DISCUSSION}

This systematic review provides an update of the evidence on courses teaching core concepts of patient safety to medical students and trainees/residents. We identified 26 studies published between January 2009 and May 2014. This is in contrast to a previous systematic review addressing the same topic but with a wider remit and time period (January 2000 to January 2009), which found 27 studies published incorporating evaluation of the interventions. ${ }^{8}$ This suggests that there is increasing interest in developing, delivering and evaluating courses teaching patient safety.

In the previously published systematic review, ${ }^{8}$ the interventions were mostly well received by participants and resulted in improvements in safety and quality knowledge scores. However, few studies were able to demonstrate changes in learners' behaviour (Kirkpatrick's level 3) or potential patient benefit (level 4b). Moreover, thematic analysis of the articles identified multiple barriers to sustainable integration of the courses, which spanned learner, faculty and institutional factors. Our systematic review has also found the included interventions to be mostly well received by participants, with improvements in safety knowledge and attitudes. Whereas more studies in our review were able to demonstrate positive changes in participant behaviour relative to the previous review, this was mainly for interventions targeted at trainees/residents rather than medical students, and most of these data on participant behaviour were self-reported. None of our identified studies demonstrated patient benefit (level 4b) from the interventions, although measurement of changes in clinical outcomes following educational interventions is notably difficult, in part due to the complexities in establishing true cause and effect.

Assessment of organisational change (level 4a) resulting from the intervention was also infrequent in our identified studies, particularly in those involving medical students. Furthermore, in the studies we reviewed, barriers to sustainable integration of the courses also spanning learner, faculty and institutional factors, were identified. Such factors included poor learner engagement, lack of expert faculty, competing educational priorities and an unsupportive institutional culture. There is no clear relationship between the length of the patient safety course and effect on learning outcomes, although a meaningful analysis of this is confounded by differences in course content and study design, quality and reporting.

Despite increasing evidence for the efficacy of educational interventions in patient safety, the wider implementation and adoption of successful interventions has been slow. ${ }^{45}{ }^{46}$ As a result, recommendations to promote curricular integration of patient safety education aim to address the barriers outlined above-for example, through investing in faculty development, promoting patient safety as a science, and integrating patient safety competencies into accreditation standards and certification examinations, to ensure protected time and incentives for medical engagement. ${ }^{4647}$

As in the earlier systematic review by Wong and colleagues, ${ }^{8}$ the majority of studies we identified in this systematic review were conducted in the US and preferentially targeted residents over medical students. The dominance of US studies in this systematic review may reflect the explicit integration of competencies in patient safety and quality improvement within national curricular statements and guidance. ${ }^{13}$ The majority of studies we identified in our review had small participant numbers, relied on single centre recruitment, and were designed as before-and-after studies with no control group or follow-up. Therefore, overall, the methodological quality of studies of patient safety interventions in medical students and trainees/residents has not changed significantly between this systematic review and the previously published one. ${ }^{8}$ This is despite recent years being characterised by the development of curricula and frameworks specifically targeting patient safety. ${ }^{12}$

Our systematic review does, however, provide some positive evidence of developments in the literature. Many of the studies we identified used previously 
published and/or validated assessment tools, demonstrating a knowledge and appreciation of the emergent evidence base in patient safety education. In line with good educational practice, the majority of studies employed experiential learning modalities (such as group discussion and project work), although one study relied solely on didactic lectures to facilitate integration into a 'busy curriculum'. ${ }^{25}$ Interestingly, case-based learning of real-life adverse events was used in few studies, despite the recognised value of reflecting and learning from error and adverse events, ${ }^{48}$ and their popularity among trainees. ${ }^{49}{ }^{50}$ It is particularly encouraging to note that we found an increase in studies explicitly commenting on sustainability of the described interventions, and their integration into the wider institution, in comparison to the previous systematic review. ${ }^{8}$ This may reflect a trend to more consideration of the longer term sustainability of patient safety interventions.

In the previous systematic review, ${ }^{8}$ the core content most commonly comprised of root cause analysis, systems thinking, general patient safety concepts and error incident reporting (all identified in over $30 \%$ of courses). In contrast, we found content to most commonly cover root cause/systems-based analysis, general patient safety concepts, communication and teamwork, quality improvement and human factors (all identified in $30 \%$ or more of published courses). Importantly, there was a marked increase in the proportion of studies covering general patient safety concepts between the previous systematic review and this one, from $34 \%$ to $65 \%$. Coverage of root cause/system-based analysis also increased from $41 \%$ to $62 \%$ of studies. In addition, between the two systematic reviews there was a decrease in the number of studies covering error/incident reporting, from $32 \%$ to $12 \%$ of studies. This discrepancy between the two systematic reviews may reflect the different search strategies used. However, it may also relate to, for example, the increasing recognition of the importance of communication and teamwork in patient safety, ${ }^{51}$ and the importance of a foundation in basic patient safety knowledge and concepts. Without sufficient studies with long-term follow-up data on patient outcomes, it is difficult to ascertain the true implications of these changes in core content. This is clearly an area for future research.

The main limitations of this systematic review relate to the quality of the included studies and the narrower focus when compared with the previous systematic review. We only included manuscripts published in the English language. We may have missed some relevant studies, although no systematic review can truly claim to find all relevant studies. There was significant heterogeneity across the studies in terms of number and type of participants targeted, the educational content of the course, the teaching methods employed, assessment tools used and the outcomes measured, which prevented a quantitative synthesis of the results. Moreover, the identification of factors influencing implementation of the courses was wholly dependent on the quality of reporting of such factors by the authors, many of whom did not stipulate identifying such factors as the primary aim of their study. It may be that important barriers and enablers to the sustainable integration of patient safety courses remain unreported, although it is important to note that we identified similar barriers and enablers to those identified in the previous systematic review. ${ }^{8}$ In box 1 we offer some recommendations for a minimum description of content that could be used in future studies evaluating patient safety courses. Adhering to these should improve study reporting and the comparison of the relative effectiveness of patient safety training interventions.

In addition to the need for future studies to address the aforementioned limitations in the evidence base, the relationship between approaches to teaching (including underpinning educational theory) and the different types of learning outcomes, should also be explored. So, too, should the relationship between implementation approaches and the impact on sustainability of an educational intervention. Such knowledge should optimise the quality of the evidence base and facilitate the development of robust evidence-based guidelines on factors that can improve outcomes at multiple levels following educational interventions for patient safety.

For those involved in medical education, there are recommendations aimed at addressing barriers to the implementation of patient safety courses. These can be classified into recommendations related to the learner, faculty, curriculum and learning environment. Learnerrelevant recommendations include: ensure courses have personal and/or clinical relevance, and offer the

Box 1 Recommendations for minimum content reporting in studies evaluating patient safety training interventions

- Study design (eg, prospective, retrospective, before and after design, control groups)

- Study setting (eg, single centre, multicentre)

- Participants including inclusion and exclusion criteria

- Intervention

- Delivery method of all aspects of the intervention (eg, online, didactic lecture, group setting)

- Thorough and explicit description of course content

- Description of those delivering the intervention (faculty), their training and their qualification

- Educational theory/theories underpinning the intervention

- Method(s) of evaluation and detailed description of exactly when these were conducted

- Specific outcomes assessed (eg, knowledge, attitudes, patient outcomes)

- Length and type of follow-up

- Data analysis methods

- Factors influencing course implementation (barriers and enablers)

- Limitations of the intervention

Areas for further work 
opportunity to apply learning to enhance engagement; ensure freedom from competing clinical/service delivery commitments; and make learning interprofessional. Faculty recommendations include: invest in faculty development; establish role models with clinical credibility; and ensure protected faculty time to deliver the patient safety course free from other commitments. Curricular recommendations include: promote patient safety as a science; avoid competing curricular demands; ensure an adequate balance between didactic and experiential learning, and between reinforcement of learning and repetition of teaching material; and provide adequate central administrative support to ensure sustainability. Finally, recommendations for the learning environment include: recognise the institutional culture as key to implementation; ensure a safe learning environment; foster links between training programmes and hospital improvement activities; and provide adequate financial support to fund the programme.

\section{CONCLUSIONS}

There is an increasing trend for the development of educational interventions in patient safety delivered to trainees/residents and medical students. The majority of such courses are well accepted by learners, and improve patient safety knowledge, skills and attitudes. Moreover, some interventions have been shown to result in positive behaviours, particularly through the subsequent engagement of trainees/residents in quality and safety improvement projects. However, no studies in the current systematic review demonstrated patient benefit. Significant methodological shortcomings in current studies exist, and additional evidence of the impact of such interventions on patient outcomes is needed. In addition, although the evidence appears to suggest some maturation in the approach and infrastructure required to support on-going delivery, significant barriers to the implementation of patient safety education remain. Further work is needed to successfully address the challenges and promote the sustainable integration of education and training in patient safety.

\section{Author affiliations \\ ${ }^{1}$ Department of Neurosurgery, Imperial College Healthcare NHS Trust, London, UK \\ ${ }^{2}$ Health Service and Population Research Department, Centre for Implementation Science, King's College London, London, UK \\ ${ }^{3}$ Department of Surgery and Cancer, Imperial College London, London, UK \\ ${ }^{4}$ Health Education North West, Manchester, UK \\ ${ }^{5}$ Department of Experimental Psychology, University of Oxford, Oxford, UK \\ ${ }^{6}$ Centre for Primary Care, NIHR School for Primary Care Research, \\ Manchester Academic Health Science Centre, University of Manchester, Manchester, UK}

Acknowledgements CV acknowledges support from the Health Foundation.

Contributors MAK participated in the article selections, conducted the literature review, helped to draft the manuscript and made subsequent revisions. MA conceived the review, conducted the literature review, participated in the design and article selections, and helped to draft the manuscript. NS and SA contributed to the article selection process and edited the manuscript for critical content. PB and CV edited the manuscript for critical content. All authors have read and approved the final manuscript.

Funding This research received no specific grant from any funding agency in the public, commercial or not-for-profit sectors.

Competing interests MAK and MA are Education Associates at the UK General Medical Council. MAK is a UK National Institute for Health Research (NIHR) Academic Clinical Fellow in Neurosurgery. NS is funded by the NIHR via the 'Collaboration for Leadership in Applied Health Research and Care, South London' at King's College Hospital NHS Foundation Trust, London, UK. NS also delivers patient safety and team interventions, and training, to hospitals internationally on a consultancy basis through London Safety and Training Solutions Ltd. SA is affiliated with the Imperial Patient Safety Translational Research Centre, which is funded by the NIHR. CV carries out occasional consultancy and advisory work on patient safety. MA is a NIHR Academic Clinical Fellow in Primary Care, and a Trustee of the Clinical Human Factors Group and has previously undertaken consultancy work for Medical Education England. MA also conducts occasional consultancy work involving faculty development for patient safety curricula delivery ('train-the-trainers' courses).

Patient consent Obtained.

Provenance and peer review Not commissioned; externally peer reviewed.

Data sharing statement No additional data are available.

Open Access This is an Open Access article distributed in accordance with the Creative Commons Attribution Non Commercial (CC BY-NC 4.0) license, which permits others to distribute, remix, adapt, build upon this work noncommercially, and license their derivative works on different terms, provided the original work is properly cited and the use is non-commercial. See: http:// creativecommons.org/licenses/by-nc/4.0/

\section{REFERENCES}

1. Association of American Medical Colleges. Report X: contemporary issues in medicine: education in safe and effective prescribing practices. Medical school objectives Project. Washington, DC Association of American Medical Colleges, 2008.

2. Association of American Medical Colleges. Report V: contemporary issues in medicine: quality of care. Medical school objectives project Washington, DC: Association of American Medical Colleges, 2001.

3. Accreditation Council for Graduate Medical Education. ACGME common program requirements. Chicago, IL: Accreditation Council for Graduate Medical Education, 2013.

4. Frank JR, Danoff D. The CanMEDS initiative: implementing an outcomes-based framework of physician competencies. Med Teach 2007;29:642-7.

5. Scheele F, Teunissen P, Van Luijk S, et al. Introducing competencybased postgraduate medical education in the Netherlands. Med Teach 2008;30:248-53.

6. World Health Organization. WHO patient safety. Curriculum guide for medical schools. Geneva, Switzerland: World Health Organization, 2009.

7. World Health Organization. WHO patient safety curriculum guide: multi-professional edition. Geneva, Switzerland: World Health Organization, 2011

8. Wong BM, Etchells EE, Kuper A, et al. Teaching quality improvement and patient safety to trainees: a systematic review. Acad Med 2010;85:1425-39.

9. Moher D, Liberati A, Tetzlaff J, et al. Preferred reporting items for systematic reviews and meta-analyses: the PRISMA statement. PLOS Med 2009;6:e1000097.

10. Coyle YM, Mercer SQ, Murphy-Cullen CL, et al. Effectiveness of a graduate medical education program for improving medical event reporting attitude and behavior. Qual Saf Health Care 2005;14:383-8.

11. Halbach JL, Sullivan LL. Teaching medical students about medical errors and patient safety: evaluation of a required curriculum. Acad Med 2005;80:600-6.

12. Madigosky WS, Headrick LA, Nelson K, et al. Changing and sustaining medical students' knowledge, skills, and attitudes about patient safety and medical fallibility. Acad Med 2006;81:94-101.

13. Patey R, Flin R, Cuthbertson $\mathrm{BH}$, et al. Patient safety: helping medical students understand error in healthcare. Qual Saf Health Care 2007;16:256-9. 
14. Bechtold ML, Scott S, Dellsperger KC, et al. Educational quality improvement report: outcomes from a revised morbidity and mortality format that emphasised patient safety. Postgrad Med $\mathrm{J}$ 2008:84:211-16.

15. Vincent C. Patient safety. Chichester: Wiley-Blackwell, 2010.

16. Hammick M, Dornan T, Steinert Y. Conducting a best evidence systematic review. Part 1: from idea to data coding. BEME Guide No. 13. Med Teach 2010;32:3-15.

17. Reed D, Price EG, Windish DM, et al. Challenges in systematic reviews of educational intervention studies. Ann Intern Med 2005;142:1080-9.

18. Anderson $\mathrm{E}$, Thorpe L, Heney $\mathrm{D}$, et al. Medical students benefit from learning about patient safety in an interprofessional team. Med Educ 2009;43:542-52.

19. Slater $B L$, Lawton $R$, Armitage $G$, et al. Training and action for patient safety: embedding interprofessional education for patient safety within an improvement methodology. $J$ Contin Educ Health Prof 2012;32:80-9.

20. Ahmed M, Arora S, Tiew S, et al. Building a safer foundation: the Lessons Learnt patient safety training programme. BMJ Qual Saf 2014;23:78-86.

21. Arora S, Sevdalis $\mathrm{N}$, Ahmed M, et al. Safety skills training for surgeons: a half-day intervention improves knowledge, attitudes and awareness of patient safety. Surgery 2012;152:26-31.

22. Jha V, Winterbottom A, Symons J, et al. Patient-led training on patient safety: a pilot study to test the feasibility and acceptability of an educational intervention. Med Teach 2013;35:e1464-71.

23. Jansma JD, Zwart DLM, Leistikow IP, et al. Do specialty registrars change their attitudes, intentions and behaviour towards reporting incidents following a patient safety course? BMC Health Serv Res 2010;10:100.

24. Jansma JD, Wagner C, Bijnen AB. Residents' intentions and actions after patient safety education. BMC Health Serv Res 2010;10:350.

25. Leung GKK, Patil NG, Ip MSM. Introducing patient safety to undergraduate medical students-a pilot program delivered by health care administrators. Med Teach 2010;32:e547-51.

26. Myung SJ, Shin J-S, Kim JH, et al. The patient safety curriculum for undergraduate medical students as a first step toward improving patient safety. J Surg Educ 2012;69:659-64.

27. Miller R, Winterton T, Hoffman WW. Building a whole new mind: an interprofessional experience in patient safety and quality improvement education using the IHI Open School. S D Med 2014;67:17-19, 21-3.

28. Cox KR, Scott SD, Hall LW, et al. Uncovering differences among health professions trainees exposed to an interprofessional patient safety curriculum. Qual Manag Health Care 2009;18:182-93.

29. Wilson AR, Fabri PJ, Wolfson J. Human error and patient safety: interdisciplinary course. Teach Learn Med 2012;24:18-25.

30. Rodrigue C, Seoane L, Gala RB, et al. Implementation of a faculty development curriculum emphasizing quality improvement and patient safety: results of a qualitative study. Ochsner $J$ 2013;13:319-21.

31. Cox LM, Logio LS. Patient safety stories: a project utilizing narratives in resident training. Acad Med 2011;86:1473-8.

32. Hall LW, Scott SD, Cox KR, et al. Effectiveness of patient safety training in equipping medical students to recognise safety hazards and propose robust interventions. Qual Saf Health Care 2010;19:3-8.
33. Jericho BG, Tassone RF, Centomani NM, et al. An assessment of an educational intervention on resident physician attitudes, knowledge, and skills related to adverse event reporting. $J$ Grad Med Educ 2010;2:188-94.

34. Dudas RA, Bundy DG, Miller MR, et al. Can teaching medical students to investigate medication errors change their attitudes towards patient safety? BMJ Qual Saf 2011;20:319-25.

35. Smith KL, Ashburn S, Rule E, et al. Residents contributing to inpatient quality: blending learning and improvement. $J$ Hosp Med 2012;7:148-53

36. Cumin D, Boyd MJ, Webster CS, et al. A systematic review of simulation for multidisciplinary team training in operating rooms. Simul Healthc 2013;8:171-9.

37. Paxton JH, Rubinfeld IS. Medical errors education: a prospective study of a new educational tool. Am J Med Qual 2010;25:135-42.

38. Stahl K, Augenstein J, Schulman $\mathrm{Cl}$, et al. Assessing the impact of teaching patient safety principles to medical students during surgical clerkships. J Surg Res 2011;170:e29-40.

39. Shaw TJ, Pernar LI, Peyre SE, et al. Impact of online education on intern behaviour around joint commission national patient safety goals: a randomised trial. BMJ Qual Saf 2012;21:819-25.

40. Scott DR, Weimer M, English C, et al. A novel approach to increase residents' involvement in reporting adverse events. Acad Med 2011;86:742-6.

41. Aboumatar HJ, Thompson D, Wu A, et al. Development and evaluation of a 3-day patient safety curriculum to advance knowledge, self-efficacy and system thinking among medical students. BMJ Qual Saf 2012;21:416-22.

42. Holland R, Meyers D, Hildebrand C, et al. Creating champions for health care quality and safety. Am J Med Qual 2010;25:102-8.

43. Gupta M, Ringer S, Tess A, et al. Developing a quality and safety curriculum for fellows: lessons learned from a neonatology fellowship program. Acad Pediatr 2014;14:47-53.

44. Tess AV, Yang JJ, Smith CC, et al. Combining clinical microsystems and an experiential quality improvement curriculum to improve residency education in internal medicine. Acad Med 2009;84:326-34.

45. Woodward HI, Mytton OT, Lemer C, et al. What have we learned about interventions to reduce medical errors? Annu Rev Public Health 2010;31:479-97.

46. Wong BM, Levinson W, Shojania KG. Quality improvement in medical education: current state and future directions. Med Educ 2012;46:107-19.

47. Pronovost PJ, Miller MR, Wachter RM, et al. Perspective: physician leadership in quality. Acad Med 2009;84:1651-6.

48. Pilpel D, Schor R, Benbassat J. Barriers to acceptance of medical error: the case for a teaching program (695). Med Educ 1998;32:3-7.

49. Fischer MA, Mazor KM, Baril J, et al. Learning from mistakes. Factors that influence how students and residents learn from medical errors. J Gen Intern Med 2006;21:419-23.

50. Teigland CL, Blasiak RC, Wilson LA, et al. Patient safety and quality improvement education: a cross-sectional study of medical students' preferences and attitudes. BMC Med Educ 2013;13:16.

51. Dedy NJ, Bonrath EM, Zevin B, et al. Teaching nontechnical skills in surgical residency: a systematic review of current approaches and outcomes. Surgery 2013;154:1000-8.

52. Carruthers S, Lawton R, Sandars J, et al. Attitudes to patient safety amongst medical students and tutors: developing a reliable and valid measure. Med Teach 2009;31:e370-6. 